\title{
Clinical features and factors for recurrence of patients receiving landiolol for atrial fibrillation after cardiac surgery
}

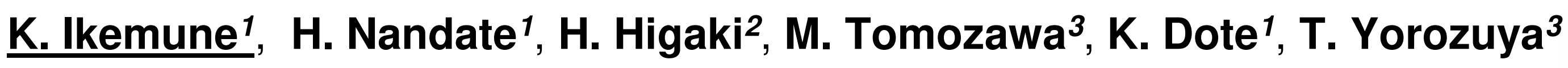 \\ 1 Intensive Care Unit, Ehime University Hospital - Ehime (Japan) \\ EHIME UNIVERSITY \\ 2 Division of Pharmacy, Ehime University Hospital - Ehime (Japan) \\ ${ }^{3}$ Department of Anesthesia and Perioperative Medicine, Ehime University Graduate School of Medicine- Ehime (Japan)
}

\section{Background:}

- Postoperative atrial fibrillation (POAF) is a common complication, and landiolol, an ultra-short acting beta-blocker, has been used to treat POAF in Japan.

- 1) In this study, we investigated the efficacy and safety of landiolol for patients with POAF after cardiac surgery.

- 2) Furthermore, since there were many cases of POAF recurrence in this study, we have also reported on the prediction factors for recurrence.

\section{Methods:}

- This retrospective study was conducted between January 2014 and September 2016.

- The study included post-cardiac surgery patients who received landiolol for POAF in the intensive care unit.

1) We first investigated the following parameters to assess the actual situation, efficacy, and safety of landiolol administration.

These included systolic blood pressure (SBP), cardiac index (Cl), and landiolol doses at the start of administration (TO) and the POAF improvement (TI). The recurrence rate of POAF were also investigated.

2) We next investigated whether $\mathrm{CHADS}_{2}(\mathbf{C 2})$ and $\mathrm{CHA}_{2} \mathrm{DS}_{2}$-VASc $(\mathbf{C 2 V})$ scores are useful as predictors of POAF recurrence.

\section{Results:}

1) Of 19 eligible patients with POAF after cardiac surgery, 18 patients $(94.7 \%)$ showed POAF improvement (Fig.1).

In these patients, landiolol was administered 1.5 [0.7-2.3] (mean [interquartile range]) $\mathrm{mcg} / \mathrm{kg} / \mathrm{min}$ at TO and 2.1[1.4-3.0] $\mathrm{mcg} / \mathrm{kg} / \mathrm{min}$ at Tl (Fig.2-1). SBP was 115[101-131] mmHg at T0 and 103 [87115] $\mathrm{mmHg}$ at $\mathrm{Tl}$ (Fig.2-2),

$\mathrm{Cl}$ was 3.0 [2.0-4.2] $\mathrm{L} / \mathrm{min} / \mathrm{m}^{2}$ at T0 and 3.0[2.63.9] L/min/ $/ \mathrm{m}^{2}$ at $\mathrm{Tl}$ (Fig.2-3), and there was no significant difference in either parameter.

2) To investigate the predictors of POAF recurrence, the 18 cases in which landiolol was effective were divided into a recurrent group (Rc, $N=10,(55.6 \%))$ and a non-recurrent group (Nrc, $N=8$ ).

C2 was 2.0 [1-3] in the Nrc group and 3.5 [2-4] in the Rc group, and was significantly different $(p=0.04)$ (Fig.3-1).

C2V was 3.5 [3-4.75] in the Nrc group and 5.5 [3-6] in the Re group ( $p=0.07$ ) (Fig.3-2).

\section{Discussion:}

1) This study showed that landiolol is effective for POAF without circulatory suppression.

2) We also examined the predictors of recurrence.

- The C2 and C2V score are known as the guide for antithrombotic therapy in patients with atrial fibrillation.

- These scores had also been reported as a predictive of POAF in after cardiac surgery ${ }^{1)}$.

- However no published studies have investigated the association between the $\mathrm{C} 2$ and $\mathrm{C} 2 \mathrm{~V}$ score in the prediction of POAF recurrence.
Fig. 1

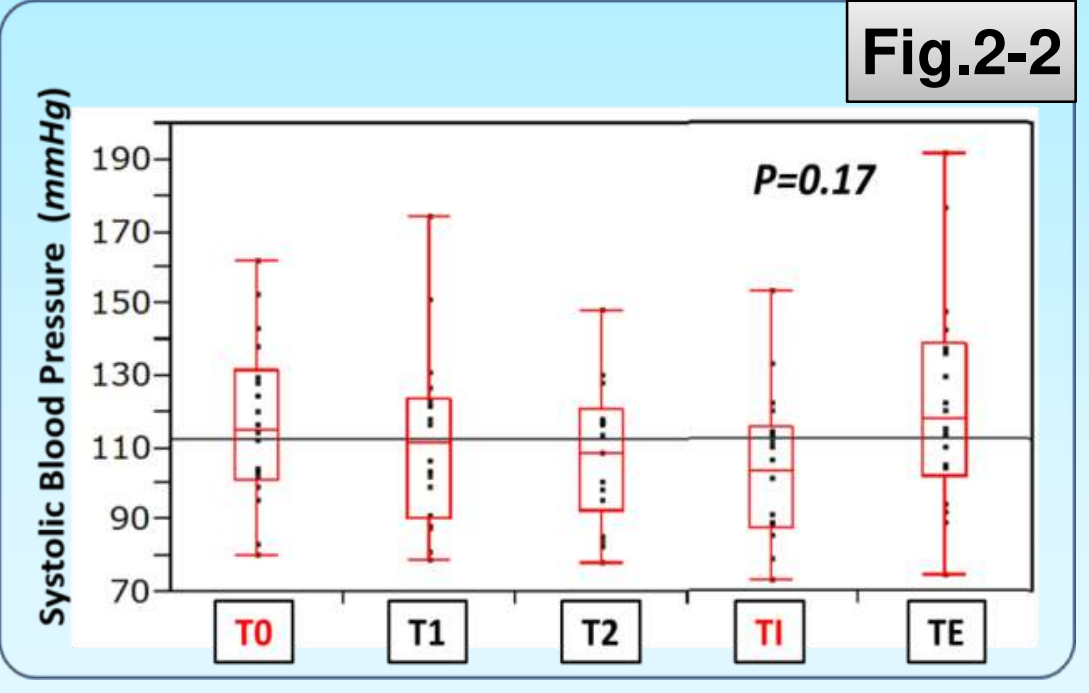

Fig.2-1

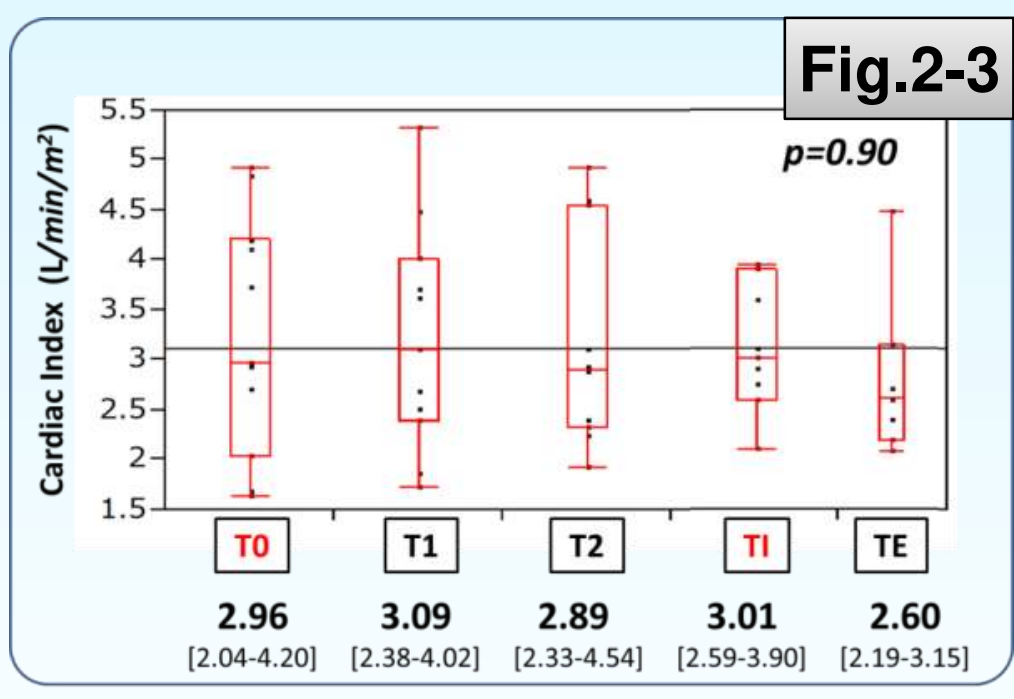

TO: at the start of administration T1: 1 hour after administration T2: 2 hours after administration TI: at the time of POAF improvement TE: at the end of administration

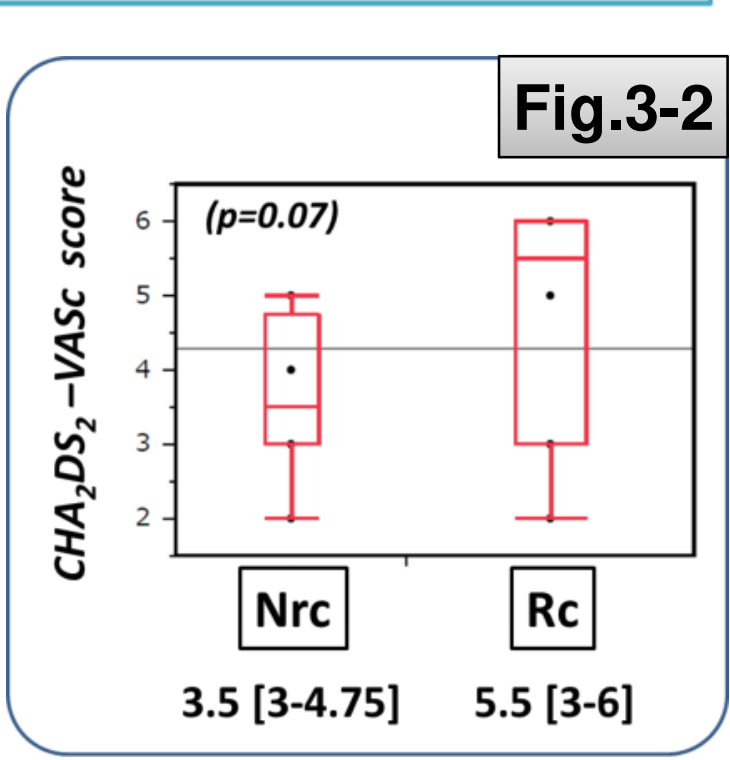

- Our results strongly suggest an association between POAF recurrence and "C2 score".

- This study is the first report to show that the $\mathbf{C 2}$ score can predict POAF recurrence after landiolol administration.

- Therefore, our results suggests that $\mathbf{C 2}$ may be used to evaluate the necessity of measures to prevent POAF recurrence.

- Moreover, from the viewpoint of simplification of management and medical economy, it can aid in predicting the appropriate withdrawal timing of landiolol. 